\title{
TITLE
}

\section{Community delivery of semi-automated fractal analysis tool in cardiac MR for trabecular phenotyping}

\section{AUTHOR LIST}

*Equally contributed

Gabriella Captur* MD PhD, ${ }^{1-3}$ Dina Radenkovic* BSc, ${ }^{1}$ Chunming Li PhD,${ }^{4,5}$ Yu Liu, ${ }^{6}$ Nay Aung MD, ${ }^{1,7}$ Filip Zemrak MD, ${ }^{1,7}$ Catalina Tobon-Gomez PhD ${ }^{8}$ Xuexin Gao, ${ }^{9}$ Perry M Elliott MBBS MD, ${ }^{1,10}$ Steffen E Petersen MD DPhil MPH, ${ }^{1,7}$ David A Bluemke MD PhD, ${ }^{11,12}$ Matthias G Friedrich MD PhD, ${ }^{13-17}$ James C Moon MB BCh $\mathrm{MD}^{1-3,10}$

1. Barts Heart Center, The Cardiovascular Magnetic Resonance Imaging Unit, St Bartholomew's Hospital, West Smithfield, London, UK

2. UCL Biological Mass Spectrometry Laboratory, Institute of Child Health and Great Ormond Street Hospital, 30 Guilford Street, London, UK

3. NIHR University College London Hospitals Biomedical Research Center, Tottenham Court Road, London, UK

4. Department of Radiology, University of Pennsylvania, Philadelphia, PA

5. School of Electronic Engineering, University of Electronic Science and Technology of China (UESTC), Chengdu P.R. China

6. College of Electronic Science and Engineering, Jilin University, Changchun, P.R.China

7. Cardiovascular Biomedical Research Unit, Barts and the London School of Medicine and Dentistry, Queen Mary University of London, London, UK

8. Division of Imaging Sciences, King's College London, London, UK

9. Circle Cardiovascular Imaging Inc, Panarctic Plaza, Calgary, Canada

10. UCL Institute of Cardiovascular Science, University College London, London, United Kingdom

11. Radiology and Imaging Sciences, Clinical Center, Center Drive, Bethesda, MA

12. Cardiovascular Imaging Department, John Hopkins Hospital, Baltimore, MA

13. Philippa \& Marvin Carsley CMR Center at the Montreal Heart Institute, Montreal, QC, Canada.

14. Department of Medicine, Heidelberg University, Heidelberg, Germany.

15. Departments of Cardiac Sciences and Radiology, University of Calgary, Calgary, AB, Canada.

16. Department of Radiology, Université de Montréal, Montreal, QC, Canada.

17. Departments of Medicine and Radiology, McGill University Health Center, Montreal, QC, Canada.

\section{CORRESPONDING AUTHOR}

Prof James C Moon

Institute of Cardiovascular Science, University College London, Gower Street, London

WC1E 6BT, UK

E-mail: j.moon@ucl.ac.uk Phone No: +44 2034563081 Fax No: +0203 4563086

\section{INSTITUTION}

UCL Institute of Cardiovascular Science, University College London, London, United Kingdom

\section{GRANT SUPPORT}

This work is supported by the UK National Institute for Health Research Rare Diseases Translational Research Collaboration (NIHR RD-TRC, \#171603), by the NIHR University College London Hospitals Biomedical Research Centre and the Biomedical Research Unit at Barts Hospital, respectively.

RUNNING TITLE Fractal analysis tool for trabecular phenotyping MANUSCRIPT TYPE Technical Development 


\section{STRUCTURED ABSTRACT}

Purpose. The trabeculae of the left ventricle vary in health and disease but their measurement is difficult. Fractal analysis of cardiac magnetic resonance (MR) images can measure trabecular complexity as a fractal dimension (FD). We report the development of easy-to-use MR fractal tools deployed on platforms accessible to all.

Materials and Methods. This HIPAA-compliant study was approved by the local institutional review board. Participants provided written informed consent. The original MATLAB implementation (region-based level set segmentation and box-counting algorithm) was recoded for two platforms (OsiriX and a clinical MR reporting platform $\left[\mathrm{cvi}^{42}\right.$, Circle Cardiovascular Imaging Inc.]). For validation 100 subjects were scanned at 1.5 Tesla and 20 imaged twice for inter-study reproducibility. Cines were analysed by the 3 tools and FD variability determined. Manual trabecular delineation by an expert reader (R1) provided ground truth contours for validation of segmentation accuracy by point-to-curve (P2C) distance estimates. Manual delineation was repeated by R1 and a second reader (R2) on 15 cases for intra/inter-observer variability.

Results. FD by OsiriX and the clinical MR reporting platform showed high correlation with MATLAB values (correlation coefficients: 0.96 [95\% CI: 0.95-0.97] and 0.96 [0.95-0.96]) and high inter-study and intra-platform reproducibility. Semi-automated contours in OsiriX and the clinical MR reporting platform were highly correlated with ground truth contours evidenced by low P2C errors: $0.882 \pm 0.76 \mathrm{~mm}$ and $0.709 \pm 0.617 \mathrm{~mm}$. Validity of ground truth contours was inferred from low P2C errors between readers (R1-R1: $0.798 \pm 0.718 \mathrm{~mm}$; R1-R2: $0.804 \pm 0.649 \mathrm{~mm})$. 
Conclusion. This set of accessible fractal tools that measure trabeculation in the heart have been validated and released to the cardiac MR community (http://j.mp/29xOw3B $)$ to encourage novel clinical applications of fractals in the cardiac imaging domain.

\section{Key words}

Etiology [16] Myocardial cardiomyopathy disease

Diagnostic testing [30] MRI

Myocardial biology [104] Structure

Word count: 2,156 


\section{TEXT}

\section{INTRODUCTION}

Abnormal and excessive trabeculation is observed across a broad spectrum of heart muscle diseases (e.g. left ventricular noncompaction $[\mathrm{LVNC}](1,2)$, hypertrophic cardiomyopathy $[\mathrm{HCM}](3)$, sickle cell anaemia(4), congenital heart disease(5)). Consequently, robust quantification of trabeculae is gaining interest for overt disease definition, e.g. LVNC(6), for tracking changes over time(7), and for detecting early disease(8).

Cine steady-state free precession (SSFP) cardiac magnetic resonance (MR) imaging is considered the modality of choice for the visualisation of trabeculae as it has unrestricted imaging planes, high spatial definition and blood:myocardial contrast, especially apically where echocardiography struggles.

Trabecular quantification is not standardized. A variety of MR-based methods have been proposed(9)) including some semi-automated approaches. However, a clinically useable technique validated against ground truths is not widely available and manual delineation by an experienced reader remains the standard approach despite being time consuming and observer dependent. To transition some of these semi-automated methods from research tools into more widely-used applications for cardiac MR, equivalent accessible software products are needed for sharing across the cardiac MR community.

We recently described a fast in-house fractal method (coded in MATLAB, The MathWorks, Inc., Natick, Massachusetts) that measures the complexity of endocardial borders as a continuous variable-the fractal dimension [FD]. Fractal analysis applied to cardiac MR by individual research groups has demonstrable utility for describing trabecular phenotypes in LVNC(2) (where the optimal diagnostic threshold for maximal apical FD was $\geq 1.3$ ), heart failure with reduced and preserved ejection fraction(10), and overt and subclinical $\operatorname{HCM}(3)$. 
Using this method, the endocardial complexity of the normal human LV has been shown to change in a characteristic pattern from base to apex, recapitulating changes along the length of the LV in the developing mouse heart(11), where the transformation from spongy to compact myocardium, coincides with the development of the coronary arteries. The fractal signature of the adult human heart has been shown to differ by ethnicity, LV mass, coexistent hypertension, and increased body mass index(6). Cardiac MR fractal analysis is currently being used to understand the FD of the adult human heart at scale, in both the UK Biobank Study $(n=100,000)$ and the US Multi-Ethnic Study of Atherosclerosis (MESA, $>3,000)$. To progress this imaging biomarker (the FD), explore its practical clinical utility in patients, ensure research transparency, mitigate against potential biases in publication, and encourage novel applications by third parties, a set of easy-to-use community-accessible fractal tools deployed on platforms familiar to cardiac imagers are needed. Such tools will allow the FD to exit expert centers allowing real world data handling of the biomarker to commence.

This technical report summarises the development, validation and release of fractal analysis tools (Figure 1) built for both an open platform, OsiriX (v5.0.1, 64-bit, Revision 10290, http://www.osirix-viewer.com, Video 1) and a clinical MR reporting platform (cvi ${ }^{42}$, Circle Cardiovascular Imaging Inc., Calgary, Canada, Video 2).

\section{MATERIALS AND METHODS}

\section{Study Participants}

Human cardiac MR studies were conducted with the approval of our local Institutional Review Board (IRB) and an ethics committee of the UK National Research Ethics Service approved the generic analysis of anonymized clinical scans. At the time of enrolment all participants gave written informed consent conforming to the declaration of Helsinki (V. revision, 2000). The authors had control of the data and information submitted for 
publication. This study conforms to the Standards for Reporting of Diagnostic Accuracy $(\mathrm{STARD})(12)$.

One-hundred individuals (aged 18 - 75 years, 100\% Caucasian) who underwent cardiac MR imaging at The Heart Hospital part of University College London Hospitals Foundation Trust, between January 2010 and February 2012. 18 Of the 23 healthy volunteers, all the LVNC cases, and 30 of the $57 \mathrm{HCM}$ participants have been previously reported $(2,3)$. These prior articles dealt with FD comparisons between health and disease whereas in this manuscript we report on the development of community accessible fractal tools, test segmentation accuracy and report on system reproducibility. Healthy volunteers had no history of cardiovascular disease or hypertension, a normal physical examination, no family history of inheritable cardiomyopathy or sudden cardiac death and no personal history of unexplained syncope. LVNC and HCM cases had been recruited from a dedicated cardiomyopathy clinic (recruitment criteria for LVNC and HCM cases and exclusion criteria for all participants were previously described(2, 3)). Twenty individuals (15 healthy volunteers, 5 LVNC) were imaged twice on two separate sessions for inter-study reproducibility.

\section{MR Imaging}

Standard clinical scans were performed using a 1.5 Tesla magnet (Avanto, Siemens Medical Solutions, Erlangen, Germany). Cardiac MR short-axis volumetric studies(13) were acquired in accordance with Society for Cardiovascular Magnetic Resonance (SCMR) guidelines, from retrospectively-gated, breath-held, balanced, cine SSFP (slice thickness, $7 \mathrm{~mm}$; interslice gap, $3 \mathrm{~mm}$; flip angle, $60-80^{\circ}$; repetition time, $3 \mathrm{~ms}$; echo time, $1.33 \mathrm{~ms}$; field of view read typically, $380 \mathrm{~mm}$; phase resolution, 75\%; typical acquired voxel size $1.5 \times 1.7 \mathrm{~mm}$; lines per segment, 12; typical temporal resolution, 25 frames). 


\section{Image Analysis}

Fractal analysis was performed as previously described(14) on the end-diastolic frames of each short-axis cine slice in the LV stack. Cardiac MR slices underwent automatic scaling according to Digital Imaging and Communications in Medicine (DICOM) pixel spacing metadata as the first image-processing step. After loading the cine stack, the graphical user interfaces (GUI) prompted the user to define the region-of-interest (ROI) using an ROI-poly tool applied to the subendocardium. Step 1 required user interaction but subsequent steps were automated and consisted of image segmentation to extract the endocardial contour followed by determination of the FD. In Step 2 a region-based level set segmentation algorithm(14) was used to extract endocardial contours before conducting box-counting fractal analysis (Step 3) as previously described(2) (Figure 2). The level set algorithm(14) is able to achieve subpixel accuracy in the extraction of the endocardial contours and deal with the intensity inhomogeneity in the images. The subpixel accuracy of the endocardial contour is necessary for the precise computation of the FD of the contours. On account of partial volume effects that tended to affect the most apical of the LV cine slices, apical endocardial contours with a bounding box global height or global width less than 49 pixels were automatically excluded from fractal analysis by the software. Results to three decimal places are automatically labelled and parsed into a text file for statistical analysis.

\section{Assessment Of Segmentation Accuracy}

Execution time for the automatic level set segmentation method in the 3 implementations (MATLAB, OsiriX, the clinical MR reporting platform) was timed and segmentation results compared with a ground truth of manual delineation, by an experienced reader $(\mathrm{R} 1,>3$ years experience in cardiac MR), blinded to other data. Manual contouring was performed in 
accordance with SCMR guidelines on standardized image interpretation and post-processing of cardiac MR(15) images except that trabeculae and papillary muscles were outlined and not included in the blood pool. Error accuracy was estimated as the mean perpendicular distance between the two sets of contour coordinates denoted as point-to-curve mean $(\mathrm{P} 2 \mathrm{C})$ error. $\mathrm{P} 2 \mathrm{C}$ errors in the basal half of the LV were also compared to the apical half (where contouring is known to be harder). Intra-observer analysis was separated by at least 7 days, and interobserver analysis was also performed (R2, 1 year experience in cardiac MR).

\section{Assessment Of Within Platform Reproducibility}

As the user interacts with the software platforms by applying the ROI-poly tool to the subendocardium, we calculated the intraobserver ( $\mathrm{R} 3$ vs R3) and interobserver (R3 vs R2) variability of FD across 30 randomly-selected scans (R3, >3 years experience in cardiac MR). Results for the original MATLAB tool were previously reported.(3)

\section{Statistical Analysis}

Statistical analysis was performed in $\mathrm{R}$ (version 3.0.1, The R Foundation for Statistical Computing). Data was curated using Research Electronic Data Capture (REDCap) infrastructure (instance: REDCap SECURE $(16)$ ). Descriptive data are expressed as mean \pm standard deviation unless otherwise stated. Distribution of data was assessed on histograms using Shapiro-Wilk test. Two-way random, single measures intraclass correlation coefficient (ICC) was used to assess reproducibility (absolute agreement) of FD calculates across platforms and between readers within platforms. The Bland-Altman method was used to estimate the mean difference (bias) and 95\% limits of agreement ( \pm 1.96 standard deviation) between paired FD measurements for each of OsiriX and the clinical MR reporting platform 
versus MATLAB. Differences in P2C errors between basal and apical sets were compared by unpaired $t$ test. A two-sided $P$ value $<0.05$ was considered significant.

\section{RESULTS}

\section{Participant Characteristics And Fractal Dimensions}

Table 1 summarizes patient baseline characteristics and FD results by the 3 methods. A whole short-axis stack fractal analysis was achievable in all 100 participants using all 3 implementations. No participant took more than 4 minutes to analyse (a typical slice takes $<10 \mathrm{~s}$ to analyse). A user manual for the OsiriX tool is provided in Supplementary Material 1. There was a high ICC for slice-specific FD by the OsiriX or clinical MR reporting platform versus MATLAB respectively: 0.96 [95\% confidence interval (CI), 0.95-0.97] and 0.96 [95\% CI, 0.95-0.96] respectively. Fractal dimension values obtained from MATLAB and the two implementations demonstrated narrow limits of agreement with minimal bias (Figure 3). There was a high ICC for inter-study slice-specific FD by MATLAB, OsiriX and the clinical MR reporting platform (respectively: 0.97 [95\% CI, 0.95-0.98], 0.96 [95\% CI, 0.94-0.97], 0.96 [95\% CI, 0.94-0.97]).

\section{Ground Truth Contours And Segmentation Accuracy}

Repeated manual delineations of trabeculae by expert readers showed low mean P2C errors: R1-R1: $0.798 \pm 0.718 \mathrm{~mm}$; R1-R2: $0.804 \pm 0.649 \mathrm{~mm}$. Expert manual delineation and the semi-automatic trabecular contours agreed well with low mean P2C errors, Table 2. The differences between manual and semi-automated contours were in the same range as intraand inter-observer manual variability $(0.882 \pm 0.76 \mathrm{~mm}$ and $0.709 \pm 0.617 \mathrm{~mm}$ for OsiriX and the clinical MR reporting platform respectively across all slices studied). Accuracy of endocardial segmentation was higher in the basal than in the apical half of the LV ( $P$ 
consistently $<0.05$ for differences, see Table 2). This trend was observed for all experiments (manual delineations as well as semi-automated segmentations) but accuracy of apical segmentation was still sufficiently accurate, with all P2C errors remaining below $1 \mathrm{~mm}$.

\section{Within Platform Reproducibility}

Intra- and inter-observer reproducibility of FD per slice was high for both platforms: OsiriX ICCs: $0.987,95 \%$ CI $0.973-0.996$ and $0.986,95 \%$ CI 0.973-0.996 respectively; clinical MR reporting platform - ICCs: $0.994,95 \%$ CI $0.992-0.996$ and 0.993 , 95\% CI $0.990-0.996$ respectively.

\section{DISCUSSION}

There is information contained in the trabecular architecture of the heart. Fractal analysis has the advantages that it measures complexity (a key biological phenomenon), is a continuous variable (like LV wall thickness or ejection fraction), and alters in health and disease. It can also be used with different imaging techniques and at different scales of observation-from adult human cardiac MR to high-resolution episcopic microscopy for development $(11,17)$. Here, the software for its analysis is shown to be easy-to-use after brief training, fast, reproducible and available, including both a free plugin for OsiriX and a plugin embedded in a widely-used clinical MR reporting platform. We commend the technique specifically to clinical researchers aiming to quantify LV trabecular complexity but emphasize that to do this, the level-set segmentation method has been designed to segment trabeculae out of the blood pool-this contrasts with other semi-automated segmentation tools recommended for standardized quantification of LV cavity size, function and mass where trabeculae and papillary muscles are sometimes included in the blood pool to improve reproducibility(18) although there is still no universal agreement regarding this issue. 
Published cardiac MR data on LV volume analyses (that exclude trabeculae) have already highlighted the better performance of basal/midventricular segmentation compared to the apex(19) (where partial volume effects are likely responsible). Relevant to the case of LV trabecular segmentation, we therefore included experiments comparing segmentation accuracy of slices in the basal LV compared to the apex. We report that whether by expert manual delineation or semi-automation, basal slices performed better than apical, but P2C errors for apical segmentation using the region-based level set method with bias field correction was still low at $<1 \mathrm{~mm}$.

Technically, we describe the 3 fractal implementations (in MATLAB, OsiriX and a clinical MR reporting platform), validated their results against ground truth contours and then applied each code to a set of clinical MR datasets demonstrating how, if caution is paid in the acquisition of the cine images, in their handling, and in the implementation of the code, these implementations can summarise LV trabecular complexity to match the standards of delineation by an expert clinical reader. These tools can now be applied to real world cardiac MR data to permit further validation and multi-center verification and it is possible that the FD of the LV may prove relevant as a clinical adjunct for the refined diagnoses of cardiomyopathy. The fractal method has already been used to describe FD ranges for healthy subjects(6): white (1.206 \pm 0.08$)$, Chinese-Americans (1.197 \pm 0.07$)$, Hispanics $(1.211 \pm$ 0.07), African-American (1.223 \pm 0.08$)$; left ventricular noncompaction $(1.392 \pm 0.01)$; heart failure with preserved and reduced ejection fraction(10) (mean FD: 1.26, 1.27 respectively); and overt hypertrophic cardiomyopathy(3) $(1.37 \pm 0.08)$.

The impact on FD of varying slice thickness(3) (6 vs. 7 vs. $8 \mathrm{~mm}$ ), magnetic field strength(8) (1.5 vs. 3 -Tesla), image sequence (fast gradient echo vs. cine SSFP) and patient-repositioning (inter-study reproducibility), has been previously reported. Future research should test the impact on FD of contrast administration, choice of phase (other than end-diastolic used so 
far) and temporal resolution, and its feasibility for right ventricular trabecular quantification. Performance of the level set segmentation has not been tested on real time cines or in the presence of arrhythmia and motion artefacts. The tools are quite generic and fractal plugins could now similarly be developed for other clinical MR reporting platforms (e.g. Medis, CMRtools). Through optimization of the level set function we believe it is plausible to expect future applications to other scenarios, including other imaging modalities (e.g. computerized tomography) and other cardiac structures (e.g. the right ventricle, atria/atrial appendage). The pursuit of such endeavors is encouraged and facilitated by products of this kind.

A fundamental question arises-how should a fractal analysis of the myocardial trabeculae be incorporated into clinical care? There are key areas to focus on. Trabecular development and the myocardial changes that take place during cardiac embryogenesis are incompletely understood processes and herein lies one potential application of fractal analysis. Abnormal trabeculae are often a disease feature (epiphenomenon) rather than a pathological entity perse(20). For example, HCM exhibits abnormal trabecular architecture and there may be a predictive role for its description in early disease(8). It may be that a high or low FD in a newly-presenting dilated cardiomyopathy patient may point to a specific genetic abnormality or prognosis, but these hypotheses are as yet unexplored. Our disease classifications are defined by our current state of knowledge, itself defined mainly by our tools and technologies and how we use them. Here cardiac MR can be a game-changer-tied to new accessible analytics it has the potential to fine-tune established disease definitions.

Limitations of this study include the reliance upon the use of Mac computers running OS X to support OsiriX, although the clinical MR reporting platform is not limited to Mac computers (albeit a commercial product). The clinical utility of cardiac MR fractal analysis in discriminating health from disease (left ventricular noncompaction, heart failure, HCM) was previously demonstrated and beyond the scope of this work where the focus was to report the 
development, validity and reproducibility, of easy-to-use and accessible MR fractal tools. For this we used 100 cardiac MR datasets (23\% healthy, 77\% participants with disease). Our analysis refers only to 2D images acquired along the long axis of the LV and future research should explore its extension to the more realistic case of 3D trabecular networks imaged by 3D whole-heart cardiac MR. Only the level set segmentation method was used-other classical image segmentation methods, such as visual thresholding, edge-detection and region-grow were not investigated on account of the several desirable advantages of the level set method discussed elsewhere(14).

In conclusion, fractal analysis of cardiac MR data measures LV endocardial complexity and provides a fractal dimension. We show that this semi-automated system, developed into accessible community tools, is able to achieve a mean point-to-curve error of less than $1 \mathrm{~mm}$ for all digital images studied. This set of accessible fractal tools that measure trabeculation in the adult human left ventricle are described and the products are now both publically (http://j.mp/29xOw3B) and commercially available. 


\section{REFERENCES}

1. Petersen SE, Selvanayagam JB, Wiesmann F, et al.: Left ventricular non-compaction: insights from cardiovascular magnetic resonance imaging. J Am Coll Cardiol 2005; 46:101105.

2. Captur G, Muthurangu V, Cook C, et al.: Quantification of left ventricular trabeculae using fractal analysis. J Cardiovasc Magn Reson 2013; 15:36.

3. Captur G, Lopes LR, Patel V, et al.: Abnormal cardiac formation in hypertrophic cardiomyopathy: fractal analysis of trabeculae and preclinical gene expression. Circ Cardiovasc Genet 2014; 7:241-248.

4. Gati S, Papadakis M, Van Niekerk N, Reed M, Yeghen T, Sharma S: Increased left ventricular trabeculation in individuals with sickle cell anaemia: physiology or pathology? Int J Cardiol 2013; 168:1658-60.

5. Madan S, Mandal S, Bost JE, et al.: Noncompaction cardiomyopathy in children with congenital heart disease: evaluation using cardiovascular magnetic resonance imaging. Pediatr Cardiol 2012; 33:215-221.

6. Captur G, Zemrak F, Muthurangu V, et al.: Fractal analysis of myocardial trabeculations in 2547 subjects: the Multi-Ethnic Study of Atherosclerosis. Radiology 2015:707-715.

7. Gati S, Papadakis M, Papamichael ND, et al.: Reversible de novo left ventricular trabeculations in pregnant women: implications for the diagnosis of left ventricular noncompaction in low risk populations. Circulation 2014:475-83.

8. Captur G, Lopes LR, Mohun TJ, et al.: Prediction of sarcomere mutations in subclinical hypertrophic cardiomyopathy. Circ Cardiovasc Imaging 2014; 7:863-867.

9. Captur G, Syrris P, Obianyo C, Limongelli G, Moon JC: Formation and malformation of cardiac trabeculae: biological basis, clinical significance, and special yield of magnetic resonance imaging in assessment. Can J Cardiol 2015; 31:1325-37. 
10. Lin LY, Su M-YM, Pham V-T, et al.: Endocardial remodeling in heart failure patients with impaired and preserved left ventricular systolic function-a magnetic resonance image study. Sci Rep 2016; 6:1-8.

11. Captur G, Wilson R, Luxán G, et al.: Morphogenesis of myocardial trabeculae in the mouse embryo. $J$ Anat 2016; 1:100-105.

12. Bossuyt PM, Reitsma JB, Bruns DE, et al.: The STARD statement for reporting studies of diagnostic accuracy: explanation and elaboration. Ann Intern Med 2003; 138:W1-12.

13. Kramer CM, Barkhausen J, Flamm SD, Kim RJ, Nagel E: Standardized cardiovascular magnetic resonance imaging (CMR) protocols, society for cardiovascular magnetic resonance: board of trustees task force on standardized protocols. J Cardiovasc Magn Reson $2008 ; 10: 35$.

14. Li C, Huang R, Ding Z, Gatenby JC, Metaxas DN, Gore JC: A level set method for image segmentation in the presence of intensity inhomogeneities with application to MRI. IEEE Trans Image Process 2011; 20:2007-16.

15. Schulz-Menger J, Bluemke DA, Bremerich J, et al.: Standardized image interpretation and post processing in cardiovascular magnetic resonance: Society for Cardiovascular Magnetic Resonance (SCMR) board of trustees task force on standardized post processing. $J$ Cardiovasc Magn Reson 2013; 15:35-54.

16. Captur G, Stables RH, Kehoe D, Deanfield J, Moon JC: Why democratize bioinformatics? BMJ Innov 2016; 129:Published Online First: 4 October 2016.

17. Captur G, Ho CY, Schlossarek S, et al.: The embryological basis of subclinical hypertrophic cardiomyopathy. Sci Rep 2016; 8:1-10.

18. Suinesiaputra A, Bluemke DA, Cowan BR, et al.: Quantification of left ventricular function and mass by cardiovascular magnetic resonance: multi-center variability and consensus contours. J Cardiovasc Magn Reson 2015; 17:63-71. 
19. Tufvesson J, Hedström E, Steding-Ehrenborg K, Carlsson M, Arheden H, Heiberg E:

Validation and development of a new automatic algorithm for time-resolved segmentation of the left ventricle in magnetic resonance imaging. Biomed Res Int 2015:1-12.

20. Arbustini E, Weidemann F, Hall JL: Left ventricular noncompaction: a distinct cardiomyopathy or a trait shared by different cardiac diseases? J Am Coll Cardiol 2014; 64:1840-1850. 


\section{TABLES}

Table 1 Characteristics of test populations and FD results.

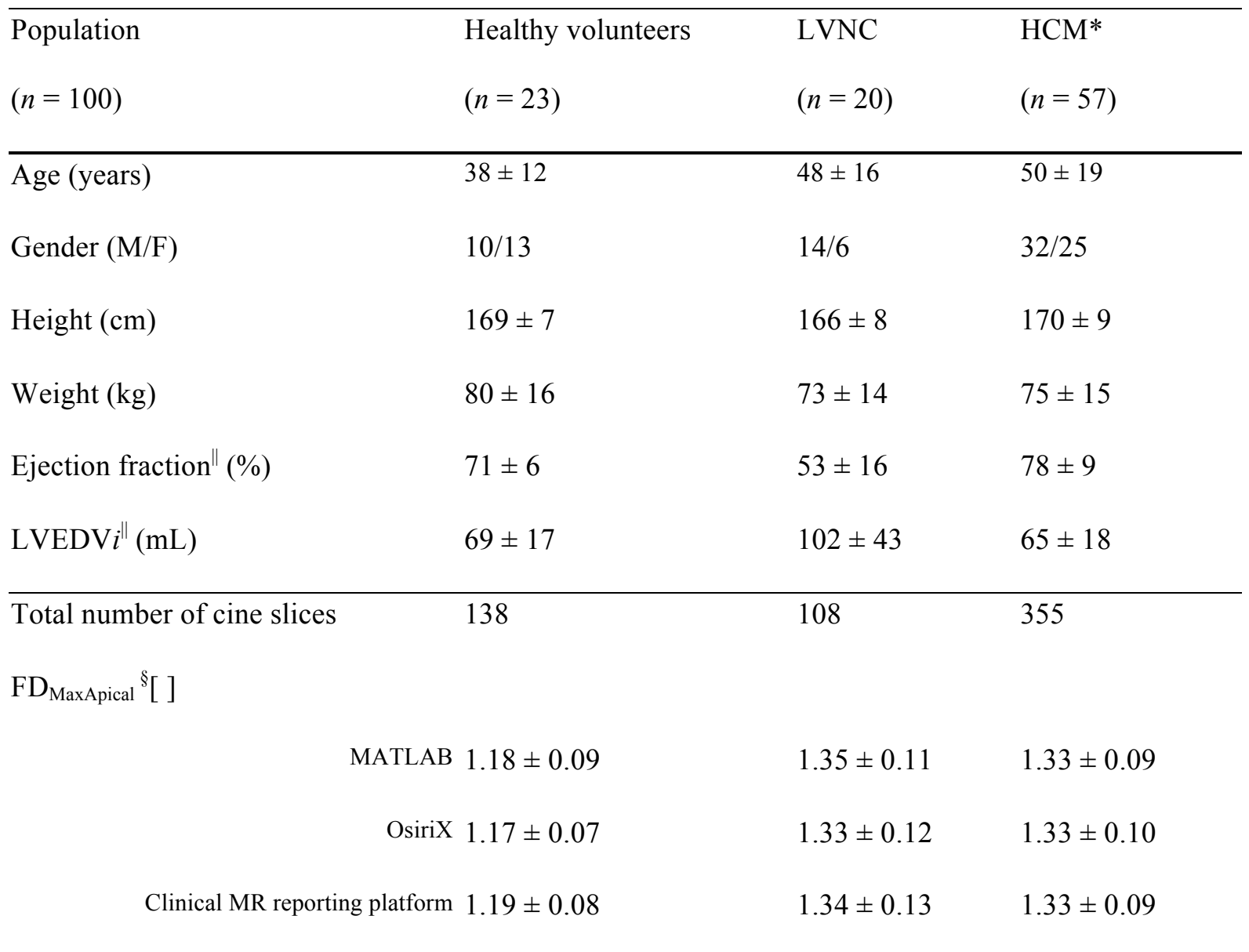

Data are presented as and mean \pm standard deviation except where otherwise stated.

* HCM population included patients with overt as well as subclinical HCM.

${ }^{\S} \mathrm{FD}_{\text {MaxApical }}$ [unitless value] was calculated as the maximal FD value in the apical half of the LV.

" Ejection fraction and LV volumes were determined in all study participants using CMRtools (CVIS, London, UK).

$\mathrm{F}=$ female HCM = hypertrophic cardiomyopathy; LVEDV $i$ = left ventricular end-diastolic volume indexed to body surface area; $\mathrm{LVNC}=$ left ventricular noncompaction; $\mathrm{M}=$ male; $\mathrm{Max}=$ maximal. 
Table 2 P2C data summarizing reproducibility of ground truths and segmentation accuracy of the two fractal tools.

\section{P2C Errors (mm)}

\begin{tabular}{|c|c|c|c|c|}
\hline \multirow[b]{2}{*}{ Slices } & \multicolumn{2}{|c|}{ Ground Truth Contours } & \multirow[b]{2}{*}{$\begin{array}{l}\text { OsiriX vs. } \\
\text { R1 Ground Truth } \\
(n=30 \text { stacks, } \\
203 \text { slices })\end{array}$} & \multirow{2}{*}{$\begin{array}{c}\text { Clinical MR reporting } \\
\text { platform vs. } \\
\text { R1 Ground Truth } \\
(n=30 \text { stacks, } \\
203 \text { slices })\end{array}$} \\
\hline & $\begin{array}{c}\mathrm{R} 1-\mathrm{R} 1 \\
(n=15 \text { stacks } \\
98 \text { slices })\end{array}$ & $\begin{array}{c}\mathrm{R} 1-\mathrm{R} 2 \\
(n=15 \text { stacks, } \\
98 \text { slices })\end{array}$ & & \\
\hline All & $0.798 \pm 0.718$ & $0.804 \pm 0.649$ & $0.882 \pm 0.760$ & $0.709 \pm 0.617$ \\
\hline Basal & $0.711 \pm 0.676\} P=0.03$ & $0.644 \pm 0.543$ & $0.819 \pm 0.698\}$ & $0.595 \pm 0.549\} P<0.001$ \\
\hline Apical & $0.935 \pm 0.803$ & $0.986 \pm 0.772$ & $0.984 \pm 0.849$ & $0.835 \pm 0.695$ \\
\hline
\end{tabular}

Data are presented as mean \pm standard deviation. For a perfect overlap between the regions P2C error should be $0 . \mathrm{P} 2 \mathrm{C}=$ point-to-curve; $\mathrm{R} 1=$ expert reader $1 ; \mathrm{R} 2=$ expert reader 2. 


\section{FIGURES}

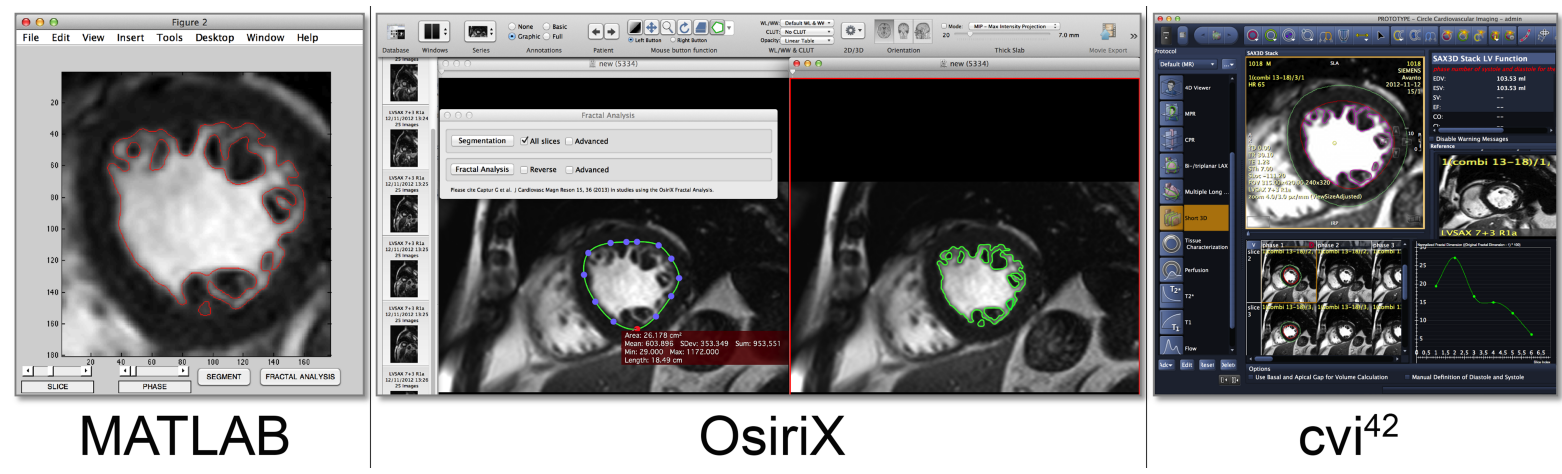

Figure 1. Community accessible fractal tools developed for cardiac magnetic resonance.

Appearance of the graphical user interfaces in the original MATLAB implementation (left) and the equivalent community-accessible tools deployed in OsiriX (middle), and the clinical MR reporting platform (right, $\mathrm{cvi}^{42}$ ). The FD is calculated for each slice in the LV short axis stack. The number of slices needed for coverage would vary depending on heart size. The most apical ventricular section was excluded from all analyses because of partial voluming effects. Maximal apical FD was derived from the apical half of the LV stack by consistently discounting the median section in unevenly numbered stacks after apical exclusion. 


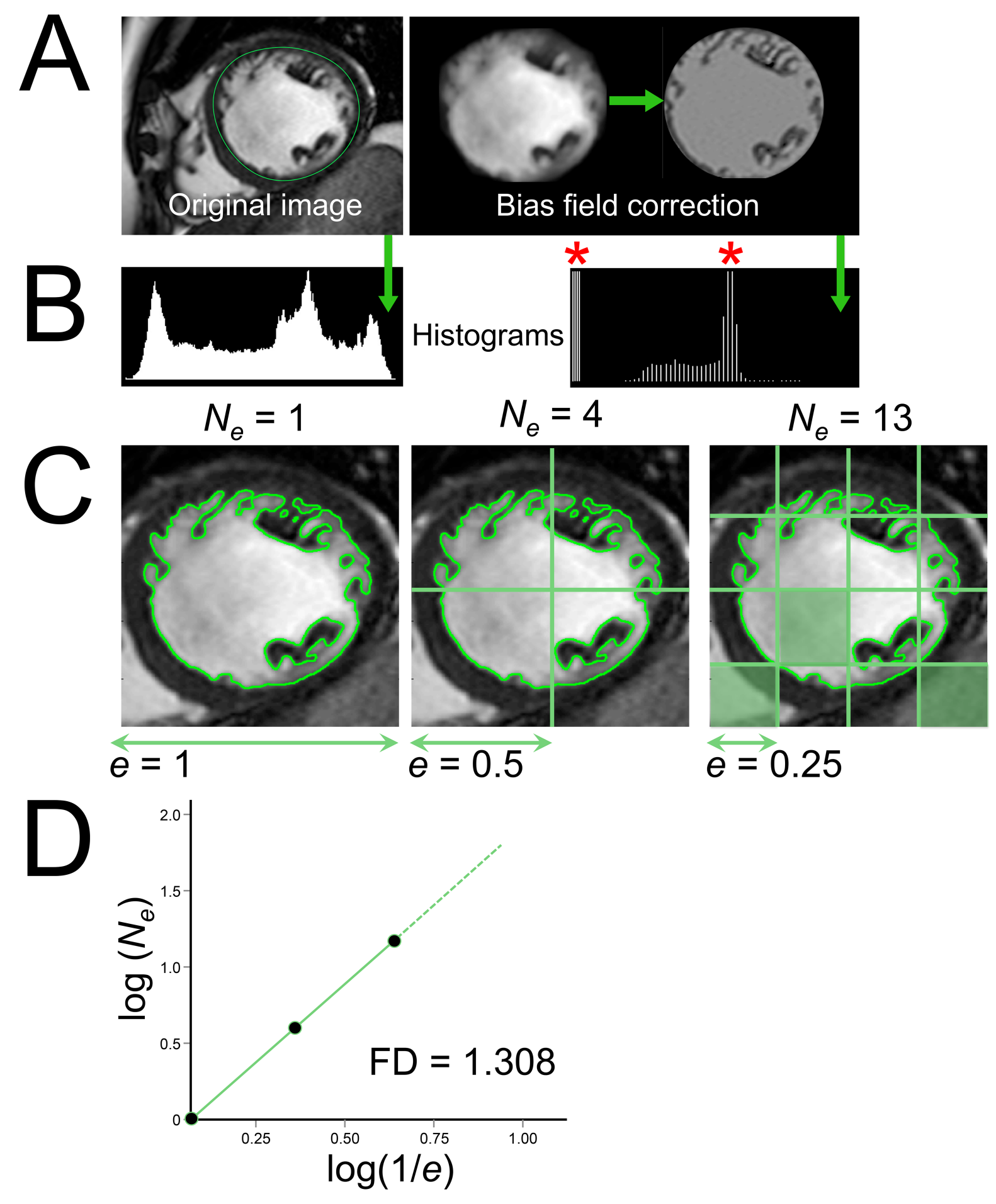

Figure 2. Region-based level set segmentation and box-counting FD applied to cardiac MR.

The only user-dependent involves placement of a ROI (in green) at the subendocardium of the original cine MR image (A, left panel) to trigger masking. All ensuing steps are automated. Pixels outside the ROI are set to zero. In the level set method contours are 
represented as the zero level set of the level set function and the segmentation problem is solved in a principled way using calculus of variations and partial differential equations. The method simultaneously estimates and corrects for any bias field across the MR image (A, right panel: seen here as an intensity inhomogeneity across the blood pool). Histograms (B) of the original images (left) and bias corrected image (right). There are 2 well-defined and well-separated peaks in the histograms of the bias corrected image (red asterisks), each corresponding to a tissue or the background in the image. The original image does not have such well-separated peaks due to the mixture of the intensity distribution caused by the bias. In the standard box-counting fractal analysis method a series of grids of boxes of ever decreasing size $(\mathbf{C})$ are applied to the digital image (only 3 grid sizes shown in this illustration). For every grid size the software counts the smallest number $(N)$ of boxes, each of side length $e$, that contain pixel detail (overlies contour pixels) and the number of pixels per box. In this pictorial representation, only 1 starting grid position and 3 box sizes are shown but the complete analysis for this slice actually involves $>50$ box sizes for each of the 4 starting grid positions. The empirical box-counting FD (1.308 in this example) is estimated from the slope of a regression line when $\log (\mathrm{Ne})$ is plotted against $\log (1 / e)$, therefore $\mathrm{FD}=$ $\log (\mathrm{Ne}) / \log (1 / e)(\mathbf{D})$. The analysis is automatically replicated on the same image 4 times, using an identical series of grid calibres but 4 different mathematically derived starting coordinates. The final FD for the contour set is computed as the mean of the 4 FDs. FD = fractal dimension; $\log =$ logarithm; ROI = region of interest. 

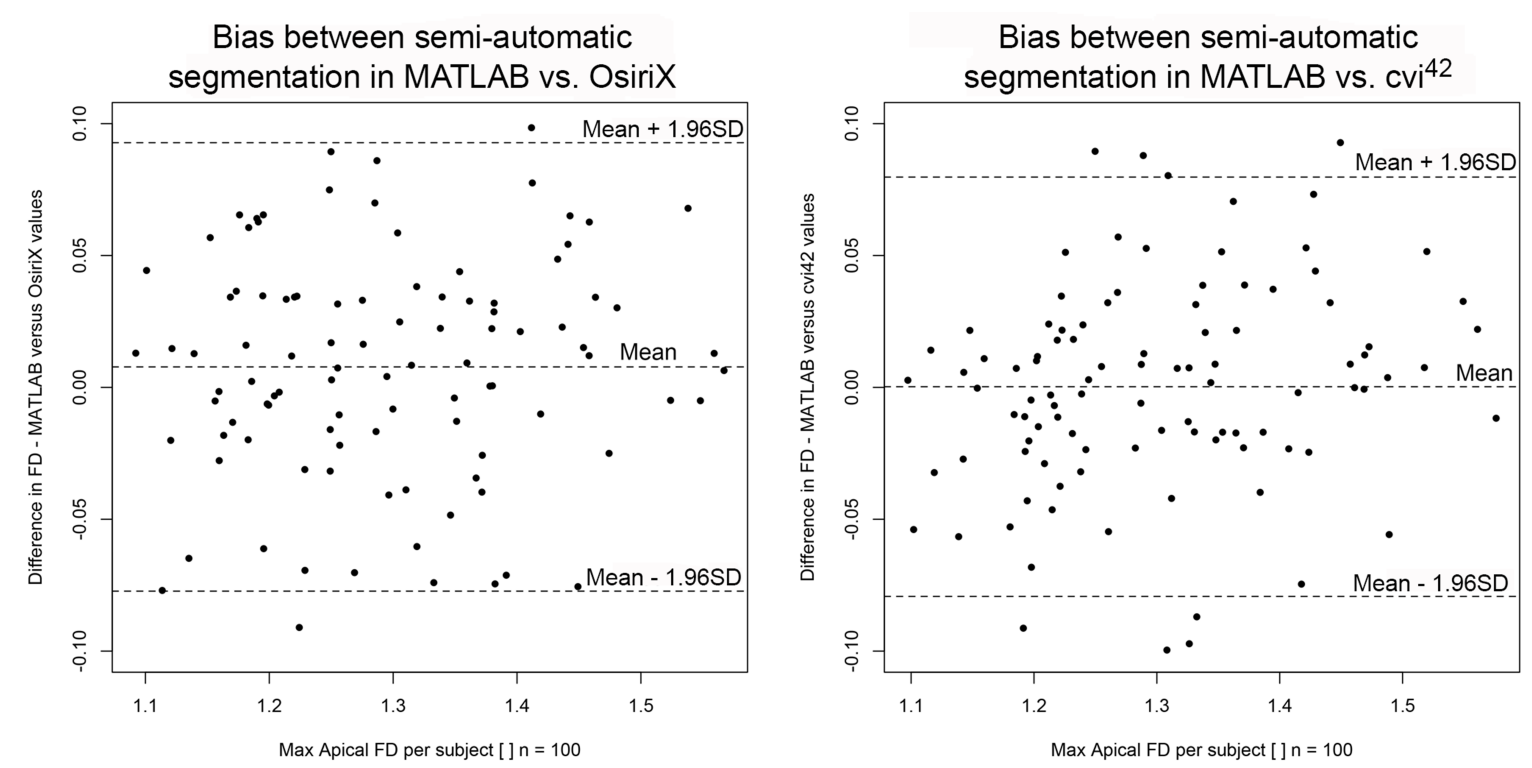

Figure 3. Bland Altman plots comparing FD results across platforms.

Differences between maximal apical FD per participant calculated by automatic segmentation in MATLAB compared to OsiriX (A) and the clinical MR reporting platform (B). Discontinuous middle line indicates mean and discontinuous horizontal lines above and below indicate mean difference $\pm 1.96 \mathrm{SD}$. The bias of each method is represented by the gap between the 'Mean' line and the $x$ axis corresponding to a zero differences. For the OsiriX method there is a positive but minimal bias of $<0.01$. FD $=$ fractal dimension; Max $=$ maximal; $\mathrm{SD}=$ standard deviation; [ ] = unitless value.

\section{VIDEOS}

Video 1. Fractal analysis using the OsiriX tool for cardiac MR.

Video 2. Fractal analysis using the tool for the clinical MR reporting platform. 